\section{Spiral Powder Overlays}

\section{P. Fraundorf and Shuhan Lin}

UM-StL Dept. of Physics \& Astronomy, and Center for

Molecular Electronics, St. Louis MO

FraundorfP@STL-MSX-NLB.umsl.edu

\section{Introduction}

Whether you encounter electron powder diffraction patterns everyday, or run across them only rarely, interpreting them may require a bit of preparation. But it's easy to lose track of the numbers for your microscope's camera constant, not to mention the bewildering array of lattice parameters and symmetries that one may encounter in the microscope. In that context, we describe overlays and diamond cubic lattices. The engaging challenge is to look for "a radial line of intersections" between the diffraction rings and the overlying spiral. When you find one that includes all lines in the diffraction pattern, you have a match "in spades"! On Figure 1 you'll find the radial line of spacing matchups for this unknown pattern marked in blue. The azimuthal position of that matchup for various elemental compounds, if one had calibrated the camera constant of the pattern precisely, is also in blue.

\section{Finding and using camera constants}

Of course it is helpful to know $\lambda \mathrm{L}$. Hence we recommend choosing a standard diffraction magnification (or camera length) and calibrating it with known specimens positioned at the standard (e.g. eucentric) height in the microscope. For example, on our Phil-

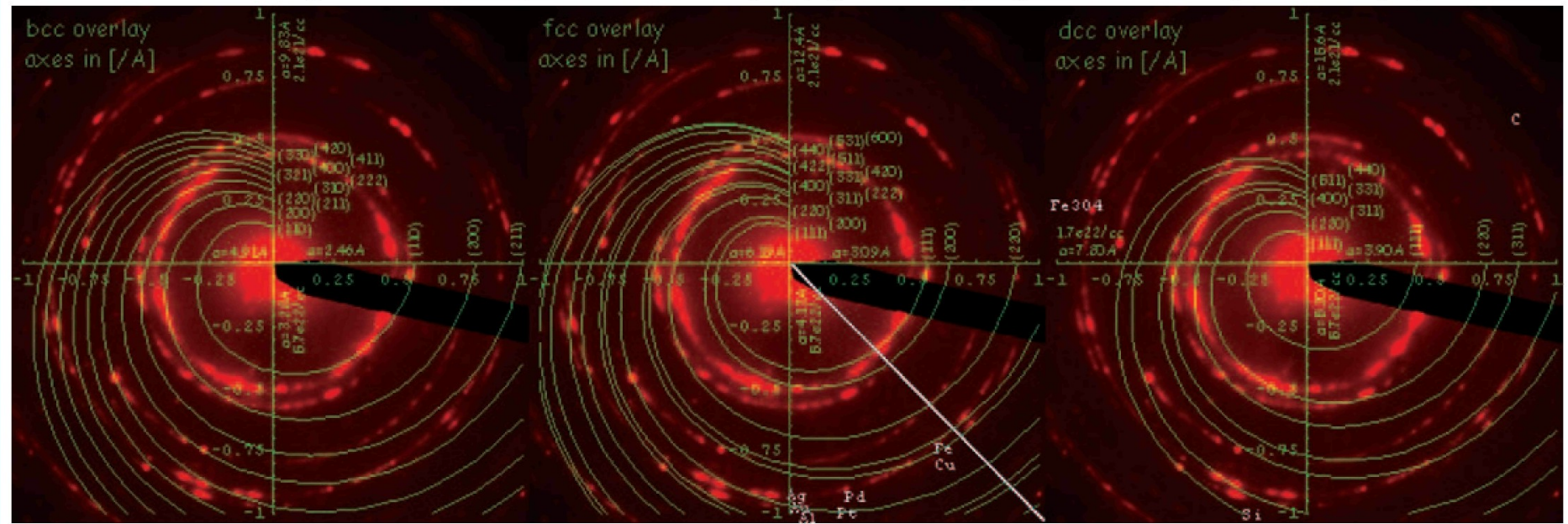

FIG. 1: Spiral body-centered, face-centered, and diamond-centered cubic overlays (green), used to identify a pattern of unknown camera constant (red) as face centered cubic (white line).

you might want to assemble for measuring camera constants, and for recognizing crystal systems most likely to yield electron powder diffraction patterns in your lab. Pulling this out of a drawer and/or computer folder thereafter might help you come up with quick answers, even if you haven't thought about analyzing diffraction data for quite a while.

To be specific, spiral overlays [1] for use with powder diffraction patterns and diffraction spacing profiles can be: (i) forgiving of uncertainties in camera constant calibration, (ii) useful for determining camera constant given patterns from an known, and (iii) sensitive, once camera constant is known, to very small differences in lattice parameter. By facilitating comparison of different lattices in a common physical metric (namely a diffraction experiment of fixed camera constant and scattering center density), their pedagogical value also extends to the comparison of crystal structures whose atomic environments share physical features (like nearest neighbor distances) in spite of major differences in symmetry. The overlays can be put onto transparencies for use directly with negatives and hardcopy, or in digital form for use with digitized images.

\section{Camera constants and their absence}

We take advantage of the fact that electron diffraction, thanks to the large size of typical lattice spacings compared to electron wavelengths, obeys the simple rule that $g_{\text {hkl }} \cong \lambda L / d_{\text {hkl. }}$. This says that lattice spacing $d_{\mathrm{hkl}}$ gives rise to a ring of radius $g_{\mathrm{hkl}}$ on a pattern of camera constant $\lambda L$. It's not always necessary to know $\lambda L$. For example, Figure 1 illustrates analysis of the experimental electron diffraction pattern from an unknown assemblage of nanocrystals (red), against overlays (green) for body-centered, face-centered, ips EM430ST, camera length $L=1200[\mathrm{~mm}]$ at $300[\mathrm{kV}]$ yields an experimentally determined value of $\lambda L$ near 23.1 [mm $\AA$ ]. Spiral overlays for a known (like that in Fig. 2 for polycrystalline Al) can, in vernier nomogram fashion, help you check the camera constant,

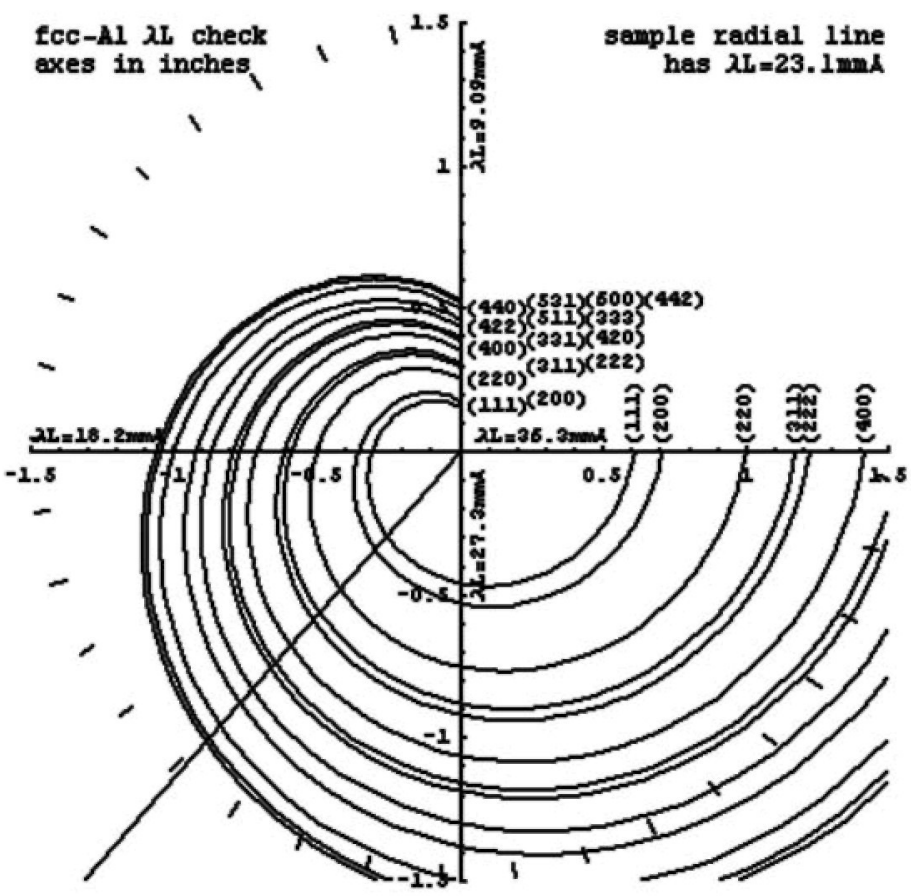

FIG. 2: An overlay designed to be used as is, or copied to a transparency with a 3-inch square axis range, for camera-constant calibration of polycrystalline aluminum electron diffraction patterns from any system. 


\section{See More. Guess Less.}
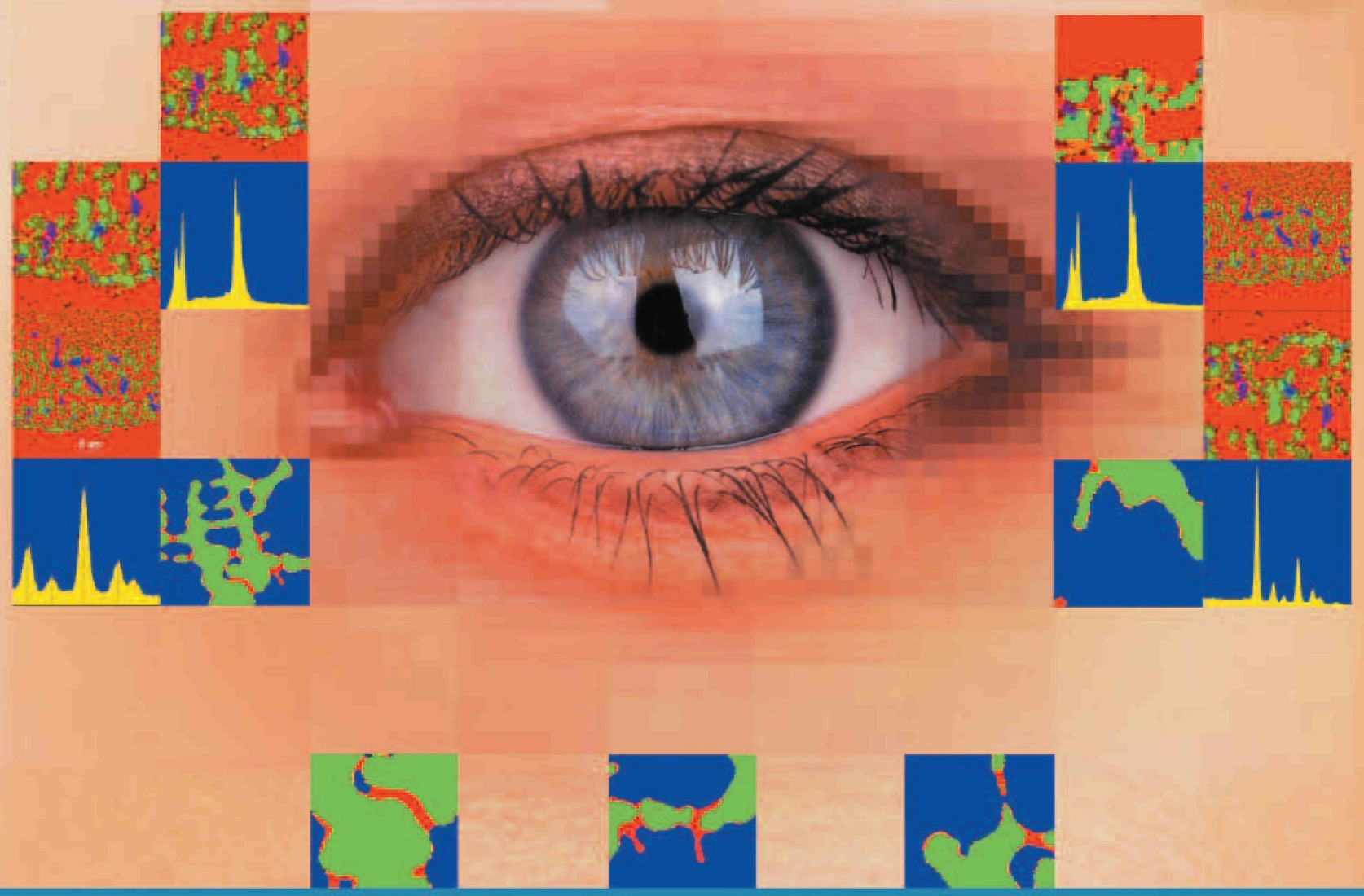

NORAN System SIX for x-ray microanalysis

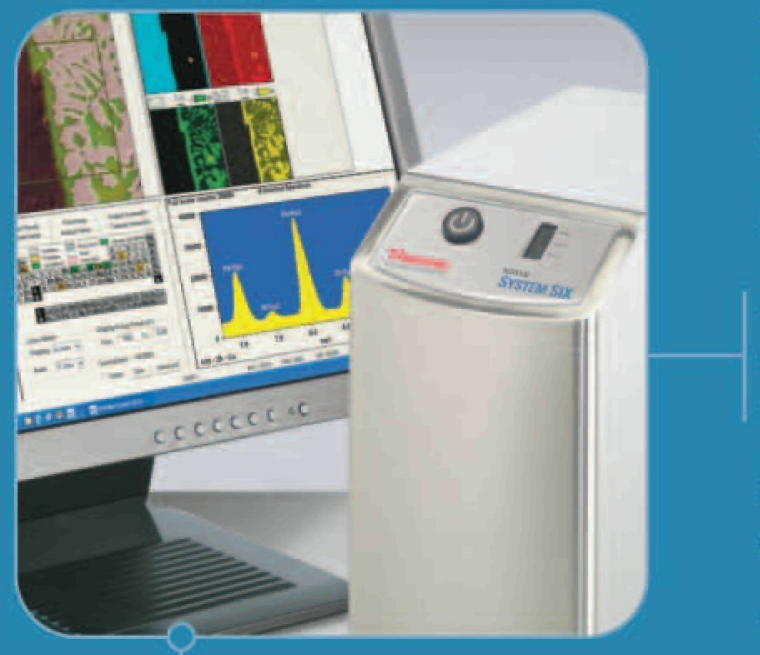

You no longer need to optimize your x-ray microanalysis parameters for just a few elements. That's because Thermo's NORAN System SIX x-ray microanalysis system gives you a complete data set with every run.

Built around our spectral imaging technology, NORAN System SIX:

- Eliminates guesswork by automatically optimizing data collection

- Gives you a full spectrum for every pixel of your electron microscope image

- Allows analysis and re-analysis of the full data set any time, anywhere.

Open your eyes to the world of NORAN System SIX at www.thermo.com/microanalysis or contact us for more information.

Telephone: 1-800-532-4752 • Email: analyze.us@thermo.com 


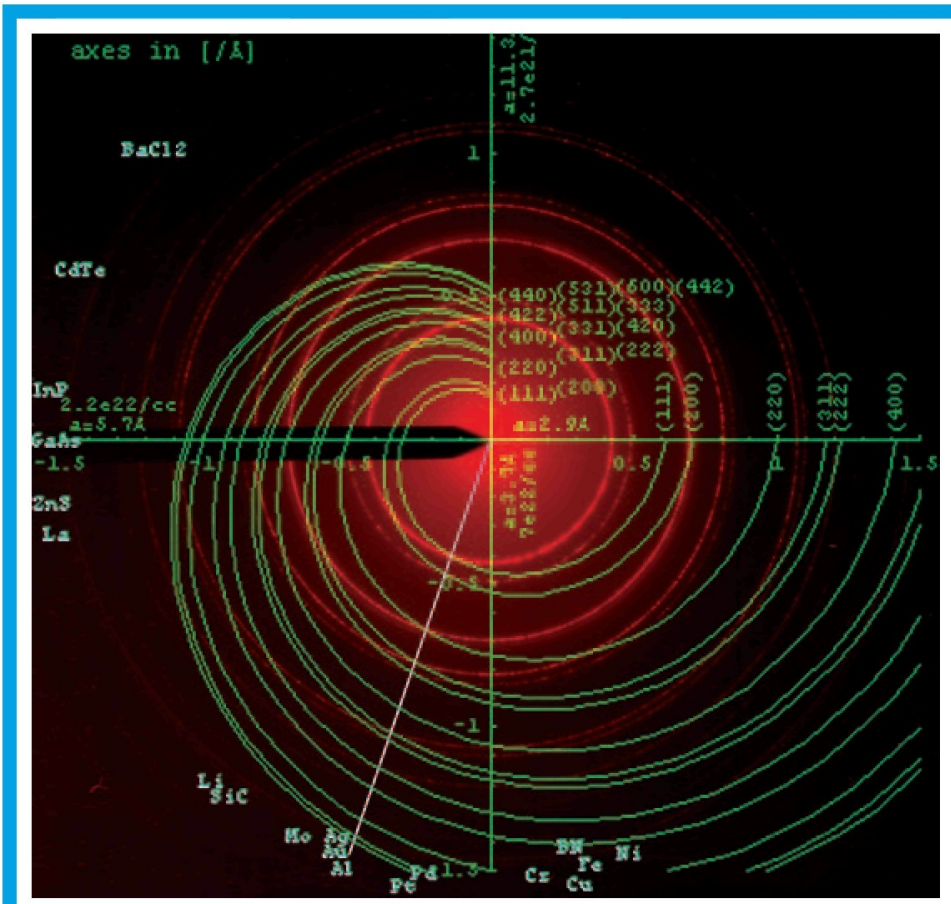

FIG. 3: Face-centered cubic overlay (green) used to distinguish Al from Ag (white line) as the source of a pattern of well known camera constant (red).

e.g. before and/or after taking data on unknowns.

If you know your camera constant precisely, spiral overlays can then help detect quite small differences in lattice parameter. For high symmetry structures where lattice parameter is indicative of atom type, this may also help determine the phase of an unknown. For example, Fig. 3 shows an f.c.c analysis of the experimental $300 \mathrm{kV}$ electron diffraction pattern from a polycrystalline $\mathrm{Al}$ thin film. In spite of the overlay's ability to reveal spacing matches even if the lattice parameter (or the camera constant of the pattern) is completely unknown, note how easily the angle of the match line for a carefully calibrated pattern allows us to distinguish between very similar lattices of $\mathrm{Al}$ and $\mathrm{Ag}$.

\section{Comparing polymorphs}

Overlays may also help examine what changes, and what doesn't change, between polymorphs of the same phase. For example, Fig. 4 shows a comparison of face centered cubic (f.c.c.) and hexagonal close packed (h.c.p.) overlays, illustrating what the associated lattices do and do not have in common. Tip: To see the fcc or hcp overlays alone, look through a pair of red-green glasses with but one eye open, so that you see only red $(f c c)$ or green $(h c p)$. Why do the diffraction spacings show so much overlap (yellow)? An atom-thick hexagonal array of atoms can be naturally stacked against a second array in one of only two ways, if the distance between nearest atoms is to be the same within and between arrays. Denote the alignment of the first array with the letter "A." The two possible adjacent layers then have " $B$ " and "C" alignments. If one stacks such layers in the sequence $\mathrm{ABCABC}$, one gets a face-centered cubic lattice (which strangely enough has four equivalent "stacking directions" along body diagonals of the cubic unit cell). Restacking of them in $A B A B A B$ form instead yields the corresponding hexagonal close-packed structure, which is not similarly isotropic (hence more spacings created than lost). In fact all f.c.c. spacings graphed are h.c.p. spacings too, except those of the form (h00).

\section{Some limitations}

The challenge of visually finding a radial line of spacingmatchups is quite a challenge for our pattern recognition skills. It may be one place where computers are still working to catch up! The "aha experience", and satisfaction of finding that match, is also hard to beat. The downside is that patterns which: (i) are spotty (too few crystals), (ii) have faint broad rings (crystals too small), or (iii) involve a mixture of lattice types, will be even more of a challenge. You may be inclined to get computer-help to sort these out. Finally, overlays for low-symmetry structures (e.g. like an olivine solid-solution series) will likely need to be generated on a case-by-case basis, i.e. as the likelihood increases of encountering each phase. This brings us to ...

\section{Do it yourself}

For creating the spirals, we use Wolfram's Mathematica's PolarPlot routine to spiral-plot a list of reciprocal lattice spacings multiplied by $\theta / 2 \pi$ (for $\theta$ values ranging from $\pi / 2$ to $2 \pi$ ) and then add to the resulting plot stuff like axes, Miller index labels, etc. The plots are monochrome images by choice, so that they stay confined to one color channel in overlays. In the examples shown here they are also binary images, although peak intensities can also be recorded in the overlays if desired.

For example, if list dlist $=\{0.486,0.793,0.930$, $1.12\}$ in $[\AA]^{-1}$ contains the first four reciprocal lattice spacings for "as small a diamond lattice cell as one is likely to encounter," then one can define $f\left[\theta_{-}\right]:=\mathrm{dli}$ ist $* \theta / 2 \pi$. After loading in Mathematica's Graphics library, the command PolarPlot $[f[\theta],\{\theta, \pi / 2,2 \pi\}, P l o t$ Range $->\{\{-$ $1.5,1.5\},\{-1.5,1.5\}\}]$ then plots spirals with axes like those above. The Show command, with some graphics primitives, allows one to add text and other markings to this plot, which after resizing to 416 pixels square yields a screen image with nice crisp lettering for cut and paste processing.

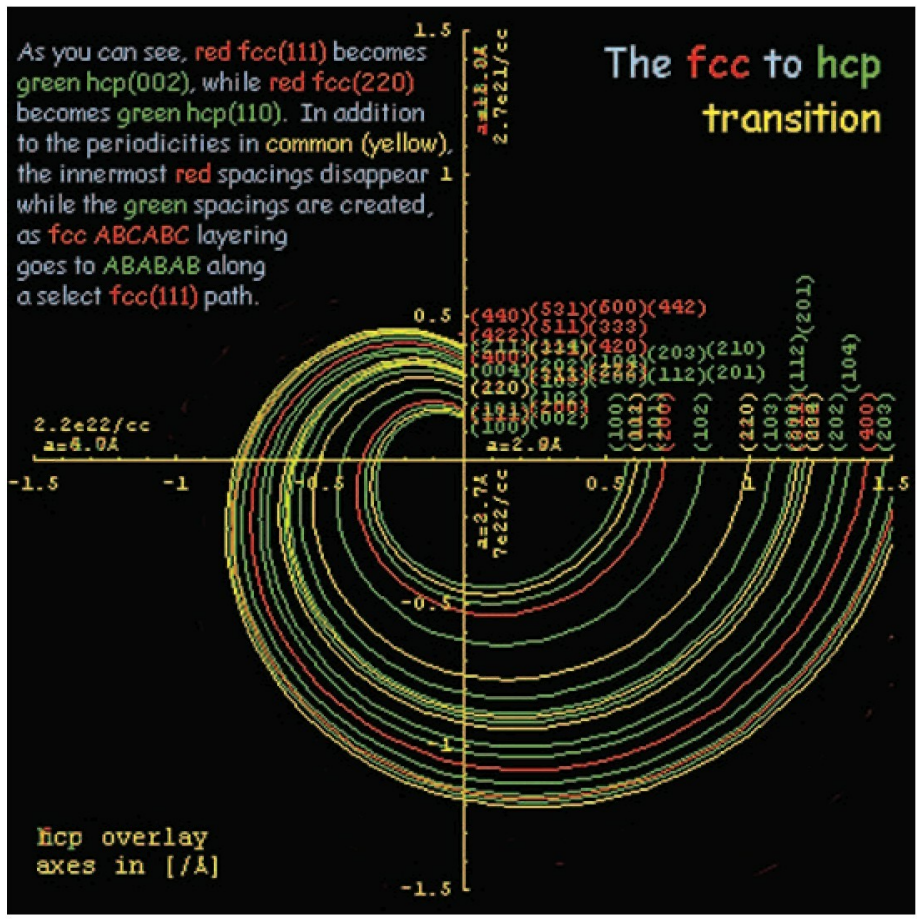

FIG. 4: Spiral overlays for face-centered cubic and hexagonal closepacked crystals with identical interatom spacing, illustrating what changes and what stays the same as the crystal re-orders from $A B C A B C$ to $A B A B A B$ layering along one of the cubic three-fold symmetry axes. 
Experimental electron diffraction patterns are set up with the usual care to area selection, specimen eucentricity, and minimal beam convergence. These are digitized either during acquisition or from photographic negatives. When the data is on film, we often digitize diffraction patterns at 600 [dpi] and $16[\mathrm{bits} / \mathrm{pixel}]$. These pattern images are then loaded into Adobe's Photoshop, and for display purposes (after contrast adjustment if appropriate) converted into 8[bit] RGB format.

A monochrome spiral overlay in negative form (e.g. white lines on black) is then copied and pasted into the experimental image's green channel. Photoshop's MoveTool then allows one to center the overlay on the pattern, typically by aligning one of the more well defined rings to the radially-symmetric tick marks on the overlay axis. Other markup information from Mathematica or elsewhere (e.g. highlighting for the radial line of intersections) may similarly be put into monochrome negative form and pasted into the blue channel. The result is a red experimental pattern with green/blue overlays, like the first three figures above.

Generating overlays specific to your applications may come in handy. Hence: (i) worksheets for making overlays to order, (ii) a 600 dpi overlay for determining the camera constant of a polycrystalline Al pattern from your microscope, (iii) overlays for fcc, bcc, hcp and dcc crystals which you may need to rescale, and (iv) possible tutorials on request, may be found on the web at http://www.umsl. edu/ fraundor/pdifovly.html .

\section{References:}

[1] P. Fraundorf and S. Lin, Proc. Microscopy and Microanalysis 2004, 1356-1357.

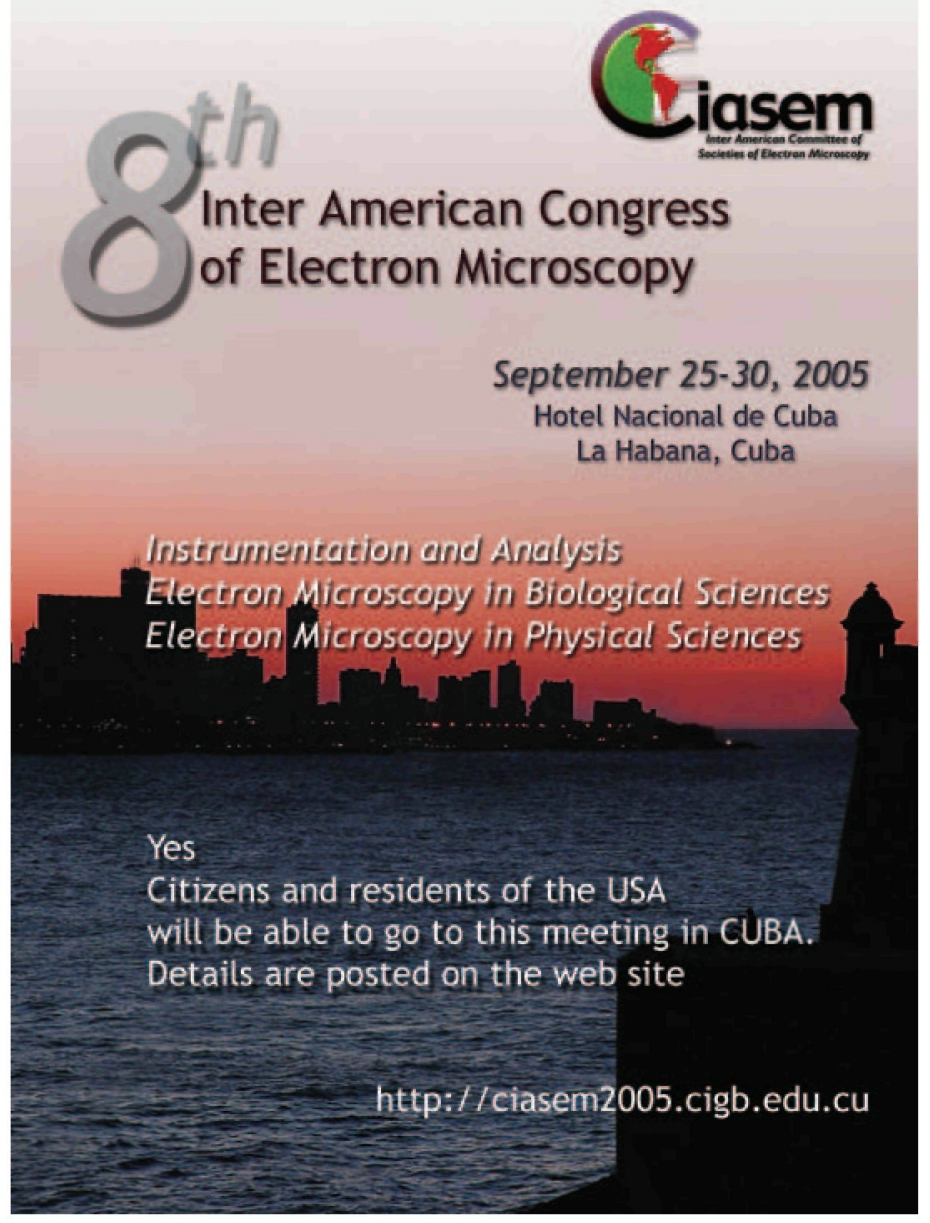

\section{Looking For More From Your SEM?}

Get it with:
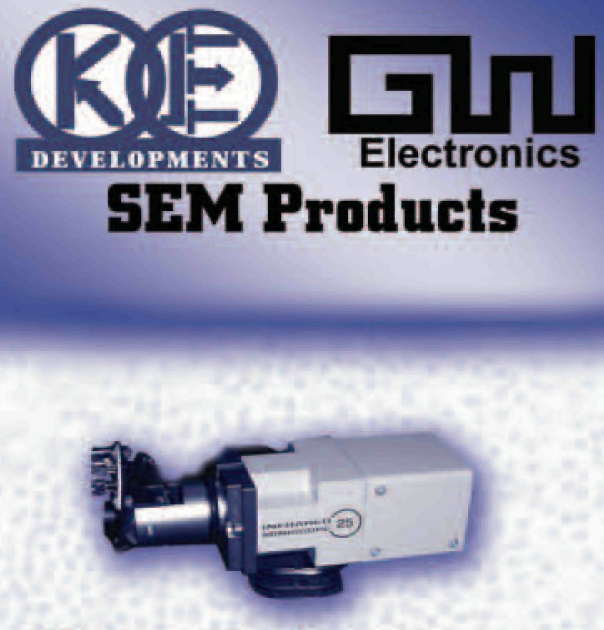

\section{Chamber Surveillance Systems}

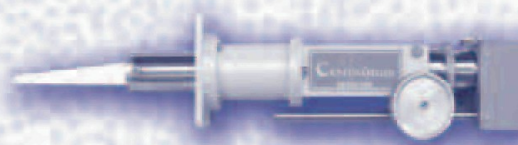

Detectors

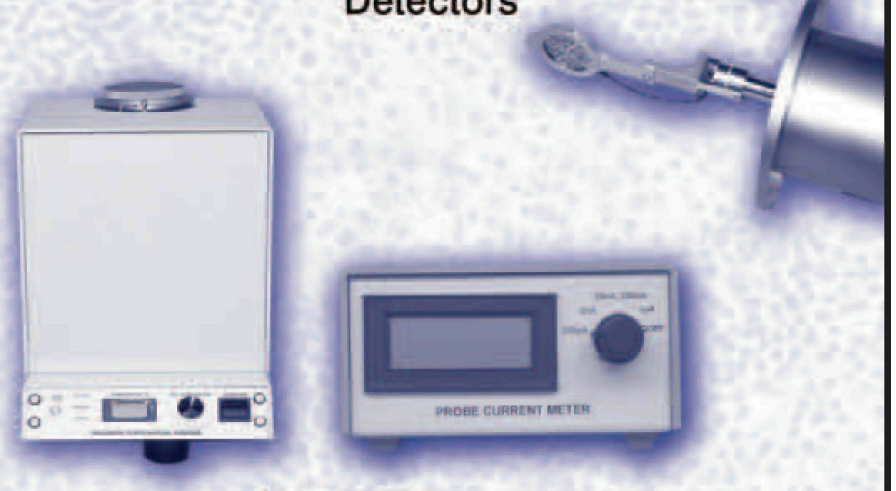

Accessories

\section{Introductory Special}

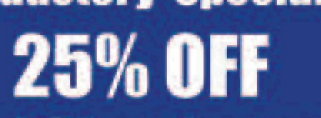

Infrared Chamberseope
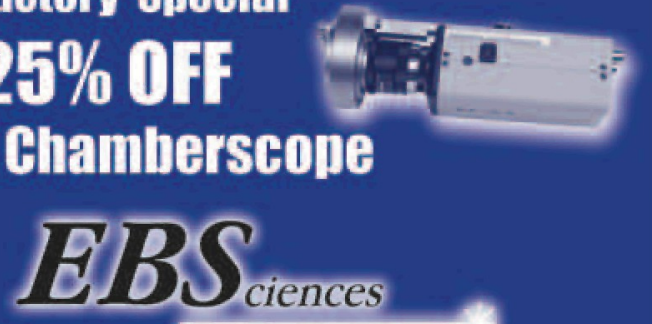

ADDING BRILIANCE TO YOUR VISION

800-992-9037 or 413-786-9322

email: ebs@ebsciences.com

www.ebsciences.com 